\title{
A Narrative Review and Expert Panel Recommendations on Dyslipidaemia Management After Acute Coronary Syndrome
}

\author{
Ashraf Reda \\ Wael Almahmeed \\ Idit Dobrecky-Mery \\ Po-Hsun Huang \\ Ursulo Juarez-Herrera \\ Naresh Ranjith \\ Tobias Sayre \\ Miguel Urina-Triana
}

\section{Video Abstract}

Keywords: Advances in Therapy, atherosclerosis, acute coronary syndrome, dyslipidemia, LDL-C, PCSK9 inhibitor, statin, cardiovascular disease, CVD, lipid-modifying therapy, LMT, guideline, barrier, awareness, low-density lipoprotein cholesterol, LDL-C, systematic review, cardiology, therapy, ezetimibe

Posted Date: September 25th, 2020

DOI: https://doi.org/10.21203/rs.3.rs-83626/v1

License: (c) (1) This work is licensed under a Creative Commons Attribution 4.0 International License.

Read Full License 


\section{Abstract}

Dyslipidemia is a major risk factor for cardiovascular disease, especially among patients who have experienced acute coronary syndrome, or ACS. Unfortunately, guidelines for managing dyslipidemia aren't always followed in practice. In a new study, an expert panel examines barriers to dyslipidemia management faced by patients and physicians outside of Western Europe and North America-and proposes ways of reducing them. Reducing low-density lipoprotein cholesterol, or LDL-C, is the primary target of lipid-modifying therapy for dyslipidemia, as it reduces the risk of cardiovascular disease through proposed mechanisms such as plaque stabilization or reduced inflammation. The American Heart Association and American College of Cardiology recommend at least a $50 \%$ reduction in LDL-C, but recommend intensification of therapy if LDL-C values remain at or above $1.8 \mathrm{mmol} / \mathrm{L}$. Meanwhile, the European Society of Cardiology and European Atherosclerosis Society recently lowered their recommended goal to $1.4 \mathrm{mmol} / \mathrm{L}$ for all patients post-ACS; that figure drops to $1.0 \mathrm{mmol} / \mathrm{L}$ for patients on maximally tolerated statins having recently experienced a second vascular event. When LDL-C goals aren't met with statins alone, ezetimibe is generally recommended as a second-line therapy-followed by PCSK9 inhibitors if a combination of statins and ezetimibe is insufficient. To understand how these guidelines are implemented outside of Western countries, the authors of the study examined 68 articles covering 17 countries of interest. They then convened a panel of seven renowned cardiologists from around the world to recommend ways of improving dyslipidemia management among patients living with ACS. Generally, their findings suggest that dyslipidemia management is often not carried out according to guideline recommendations, especially among high-risk patients. Results show that LDL-C control after ACS remains suboptimal in these countries. Rates of LDL-C goal achievement reported 1 month after ACS ranged from approximately 56 to $84 \%$. Most patients were treated with a statin after ACS, and patients on intensive lipid-modifying therapy were more likely to achieve their LDL-C goal. But target attainment rates were still suboptimal--approximately $57 \%$ for patients in Hong Kong according to one study. The low rates of use reported for high-potency statin and ezetimibe suggest that lipidmodifying therapy is often not intensified in clinical practice. The expert panel offers four recommendations for improving these shortfalls. The panel supports recent guideline updates that recommend lowering LDL-C to less than $1.4 \mathrm{mmol} / \mathrm{L}$ post-ACS. Additionally, all patients with ACS should receive a high-intensity statin therapy, if tolerated, regardless of their baseline level. The panel also recommends improving three important facets of dyslipidemia management: tailored treatment, outpatient follow-up, and education, as well as, prioritising dyslipidaemia management within healthcare policy. Country-specific treatment algorithms should be developed to address differences in healthcare systems and the availability of newer therapies. And outpatient follow-up should be arranged prior to discharge and scheduled regularly in the months that follow. Additionally, patients should be educated on LDL-C goals before discharge and during follow-up. Understanding where they're headed could motivate patients to stay the course with lipid-modifying therapy post-ACS. 